\title{
Existence of solutions for impulsive fractional boundary value problems via variational method
}

\author{
Guoqing Chai* and Jinghua Chen
}

\section{"Correspondence:}

mathchgq@163.com

College of Mathematics and

Statistics, Hubei Normal University,

Huangshi, Hubei 435002, P.R. China

\section{Springer}

$$
\begin{aligned}
& \text { Abstract } \\
& \text { In this paper, the authors consider the following fractional boundary value problem } \\
& \text { for impulsive fractional differential equations: } \\
& \qquad\left\{\begin{array}{l}
{ }_{t} D_{T}^{\alpha}\left({ }_{0}^{c} D_{t}^{\alpha} u(t)\right)+a(t) u(t)=f\left(t, u(t),{ }_{0}^{c} D_{t}^{\alpha} u(t)\right), \quad t \neq t t_{j} \text {, a.e. } t \in[0, T], \\
\Delta\left({ }_{t} D_{T}^{\alpha-1}\left({ }_{0}^{c} D_{t}^{\alpha} u\right)\right)\left(t_{j}\right)=l_{j}\left(u\left(t_{j}\right)\right), \quad j=1,2, \ldots, n, \\
u(0)=u(T)=0,
\end{array}\right. \\
& \text { where } \alpha \in(1 / 2,1], 0=t_{0}<t_{1}<t_{2}<\cdots<t_{n}<t_{n+1}=T, f:[0, T] \times \mathbb{R} \times \mathbb{R} \rightarrow \mathbb{R} \text { and } \\
& I_{j}: \mathbb{R} \rightarrow \mathbb{R}, j=1,2, \ldots, n, \text { are continuous functions, } a \in C([0, T]) \text { and }
\end{aligned}
$$

By using the variational method and iterative technique, the authors show the existence of at least one nontrivial solution to the above boundary value problem.

Keywords: fractional differential equations; critical point theory; variational method; impulsive equation; iterative technique

\section{Introduction}

Fractional calculus has applications in many areas including fluid flow, electrical networks, probability and statistics, chemical physics and signal processing, etc. For details, see [1-6] and the references therein. In recent years, there are many papers dealing with the existence of solutions of nonlinear initial (or boundary) value problems of fractional equations by applying nonlinear analysis such as fixed point theorems, lower and upper solutions method, monotone iterative method, coincidence degree theory. However, up to now, there are few results on the solutions to fractional boundary value problems that are established by the variational methods; see, for example, [7-18]. It is often very difficult to establish a suitable space and variational functional for fractional boundary value problem, especially for the fractional equations including both left and right fractional derivatives.

(c) The Author(s) 2017. This article is distributed under the terms of the Creative Commons Attribution 4.0 International License (http://creativecommons.org/licenses/by/4.0/), which permits unrestricted use, distribution, and reproduction in any medium, provided you give appropriate credit to the original author(s) and the source, provide a link to the Creative Commons license, and indicate if changes were made. 
For the first time, Jiao and Zhou [7] showed that the critical point theory is an effective approach to tracking the existence of solutions to the following fractional boundary value problem (BVP for short):

$$
\left\{\begin{array}{l}
{ }_{t} D_{T}^{\alpha}\left({ }_{0} D_{t}^{\alpha} u(t)\right)=\nabla F(t, u(t)), \quad \text { a.e. } t \in[0,1] \\
u(0)=u(T)=0
\end{array}\right.
$$

From then on, problem (1.1) and its related forms have been further studied by researchers, see, for example, [8-18], and interesting results on the existence of solutions, such as one nontrivial solution, three solutions or infinitely many solutions, were obtained by using the variational methods and the critical point theory.

On the other hand, impulsive boundary value problems for differential equations were intensively studied by topological methods over the past decade. Such problems appear in mathematical models with sudden changes of their states in population dynamics, pharmacology, optimal control, etc. [19]. The existence of solutions of impulsive problems was also treated by the variational methods and critical point theorems (see [20-22]). The pioneering work in this direction is the paper of Nieto and O'Regan [23], where the secondorder impulsive problem

$$
\left\{\begin{array}{l}
-u^{\prime \prime}+\lambda u=f(t, u), \quad t \neq t_{j}, \text { a.e. } t \in[0, T] \\
\Delta u^{\prime}\left(t_{j}\right)=I_{j}\left(u\left(t_{j}\right)\right), \quad j=1,2, \ldots, n \\
u(0)=u(T),
\end{array}\right.
$$

is studied by the minimization and the mountain pass theorem.

Investigating the impulsive problems for fractional equations via variational method is interesting. Recently, Bonanno et al. [16] and Rodrínguez-López and Tersian [17] first studied the following Dirichlet boundary value problem for fractional differential equation with impulsive effects:

$$
\left\{\begin{array}{l}
{ }_{t} D_{T}^{\alpha}\left({ }_{0}^{c} D_{t}^{\alpha} u(t)\right)+a(t) u(t)=\lambda f(t, u(t)), \quad t \neq t_{j}, \text { a.e. } t \in[0, T] \\
\Delta\left({ }_{t} D_{T}^{\alpha-1}\left({ }_{0}^{c} D_{t}^{\alpha} u\right)\right)\left(t_{j}\right)=\mu I_{j}\left(u\left(t_{j}\right)\right), \quad j=1,2, \ldots, n, \\
u(0)=u(T)=0,
\end{array}\right.
$$

where $\alpha \in(1 / 2,1], 0=t_{0}<t_{1}<t_{2}<\cdots<t_{n}<t_{n+1}=T, f, I_{j}$ and $a$ are continuous functions. Under the condition $0<a_{1} \leq a(t) \leq a_{2}$, the authors obtained the existence results of at least one solution or three solutions by using the minimization and three critical point theorem.

More recently, Nyamoradi et al. [18] investigated the following impulsive fractional boundary problem:

$$
\left\{\begin{array}{l}
{ }_{t} D_{T}^{\alpha}\left({ }_{0}^{c} D_{t}^{\alpha} u(t)\right)+a(t) u(t)=f(t, u(t)), \quad t \neq t_{j}, \text { a.e. } t \in[0, T] \\
\Delta\left({ }_{t} D_{T}^{\alpha-1}\left({ }_{0}^{c} D_{t}^{\alpha} u\right)\right)\left(t_{j}\right)=I_{j}\left(u\left(t_{j}\right)\right), \quad j=1,2, \ldots, n, \\
u(0)=u(T)=0
\end{array}\right.
$$

where $\alpha \in(1 / 2,1], 0=t_{0}<t_{1}<t_{2}<\cdots<t_{n}<t_{n+1}=T, f:[0, T] \times \mathbb{R} \times \mathbb{R} \rightarrow \mathbb{R}$ and $I_{j}: \mathbb{R} \rightarrow \mathbb{R}, j=1,2, \ldots, n$, are continuous functions, $a \in C([0, T])$. Under the condition 
$\operatorname{essinf}_{t \in[0, T]} a(t)=m>-\lambda_{1}$, the author proved the existence of at least one solution or infinitely many solutions by using critical point theory and variational methods.

In this paper, the authors consider the following fractional boundary value problem for impulsive fractional differential equations:

$$
\left\{\begin{array}{l}
{ }_{t} D_{T}^{\alpha}\left({ }_{0}^{c} D_{t}^{\alpha} u(t)\right)+a(t) u(t)=f\left(t, u(t),{ }_{0}^{c} D_{t}^{\alpha} u(t)\right), \quad t \neq t_{j}, \text { a.e. } t \in[0, T] \\
\Delta\left({ }_{t} D_{T}^{\alpha-1}\left({ }_{0}^{c} D_{t}^{\alpha} u\right)\right)\left(t_{j}\right)=I_{j}\left(u\left(t_{j}\right)\right), \quad j=1,2, \ldots, n, \\
u(0)=u(T)=0
\end{array}\right.
$$

where $\alpha \in(1 / 2,1], 0=t_{0}<t_{1}<t_{2}<\cdots<t_{n}<t_{n+1}=T, f:[0, T] \times \mathbb{R} \times \mathbb{R} \rightarrow \mathbb{R}$ and $I_{j}: \mathbb{R} \rightarrow \mathbb{R}$, $j=1,2, \ldots, n$, are continuous functions, $a \in C([0, T])$ and

$$
\begin{aligned}
& \Delta\left({ }_{t} D_{T}^{\alpha-1}\left({ }_{0}^{c} D_{t}^{\alpha} u\right)\right)\left(t_{j}\right)={ }_{t} D_{T}^{\alpha-1}\left({ }_{0}^{c} D_{t}^{\alpha} u\right)\left(t_{j}^{+}\right)-{ }_{t} D_{T}^{\alpha-1}\left({ }_{0}^{c} D_{t}^{\alpha} u\right)\left(t_{j}^{-}\right), \\
& { }_{t} D_{T}^{\alpha-1}\left({ }_{0}^{c} D_{t}^{\alpha} u\right)\left(t_{j}^{+}\right)=\lim _{t \rightarrow t_{j}^{+}} D_{T}^{\alpha-1}\left({ }_{0}^{c} D_{t}^{\alpha} u\right)(t), \quad{ }_{t} D_{T}^{\alpha-1}\left({ }_{0}^{c} D_{t}^{\alpha} u\right)\left(t_{j}^{-}\right)=\lim _{t \rightarrow t_{j}^{t}} D_{T}^{\alpha-1}\left({ }_{0}^{c} D_{t}^{\alpha} u\right)(t) .
\end{aligned}
$$

Owing to the occurrence of the fractional derivative ${ }_{0}^{c} D_{t}^{\alpha} u(t)$ included in the function $f$, the BVP (1.2) is not variational and it is unable to dealt with (1.2) directly as in [16-18] by constructing some functional $\varphi$ such that its critical point is exactly the solution to BVP (1.2). To overcome the difficulty appearing here, we shall apply the iterative technique combined with the variational method to BVP (1.2). Roughly speaking, for a certain $u_{1}$, consider the following BVP:

$$
\left\{\begin{array}{l}
{ }_{t} D_{T}^{\alpha}\left({ }_{0}^{c} D_{t}^{\alpha} u(t)\right)+a(t) u(t)=f\left(t, u(t),{ }_{0}^{c} D_{t}^{\alpha} u_{1}(t)\right), \quad t \neq t_{j}, \text { a.e. } t \in[0, T], \\
\Delta\left({ }_{t} D_{T}^{\alpha-1}\left({ }_{0}^{c} D_{t}^{\alpha} u\right)\right)\left(t_{j}\right)=I_{j}\left(u\left(t_{j}\right)\right), \quad j=1,2, \ldots, n, \\
u(0)=u(T)=0,
\end{array}\right.
$$

by using the mountain pass theorem, we can obtain one solution $u_{2}$ corresponding to the above BVP. Repeating this step, we will find a sequence $\left\{u_{n}\right\}$, which will converge to a solution of BVP (1.2).

The paper is arranged as follows. In Section 2, the authors present some necessary preliminary facts that will be needed in the paper. In Section 3, the authors establish the existence of nontrivial solutions for BVP (1.2) and give one example to show the effectiveness of the result obtained.

\section{Preliminaries}

To apply the variational method with the iterative technique to the existence of solutions for BVP (1.2), we shall state some basic notations and results, which will be used in the proof of our main result.

Definition 2.1 ([6]) Let $f$ be a function defined on $[a, b]$. The left and right RiemannLiouville fractional integrals of order $\gamma$ for function $f$ denoted by ${ }_{a} D_{t}^{-\gamma} f(t)$ and ${ }_{t} D_{b}^{-\gamma} f(t)$, respectively, are defined by

$$
{ }_{a} D_{t}^{-\gamma} f(t)=\frac{1}{\Gamma(\gamma)} \int_{a}^{t}(t-s)^{\gamma-1} f(s) d s, \quad t \in[a, b], \gamma>0,
$$


and

$$
{ }_{t} D_{b}^{-\gamma} f(t)=\frac{1}{\Gamma(\gamma)} \int_{t}^{b}(s-t)^{\gamma-1} f(s) d s, \quad t \in[a, b], \gamma>0,
$$

provided in both cases that the right-hand side is pointwise defined on $[a, b]$, where $\Gamma$ is the gamma function.

Definition 2.2 ([6]) Let $f$ be a function defined on $[a, b]$. The left and right RiemannLiouville fractional derivatives of order $\gamma$ for function $f$ denoted by ${ }_{a} D_{t}^{\gamma} f(t)$ and ${ }_{t} D_{b}^{\gamma} f(t)$, respectively, exist almost everywhere on $[a, b] \cdot{ }_{a} D_{t}^{\gamma} f(t)$ and ${ }_{t} D_{b}^{\gamma} f(t)$ are represented by

$$
{ }_{a} D_{t}^{\gamma} f(t)=\frac{1}{\Gamma(n-\gamma)} \frac{d^{n}}{d t^{n}} \int_{a}^{t}(t-s)^{n-\gamma-1} f(s) d s, \quad t \in[a, b],
$$

and

$$
{ }_{t} D_{b}^{\gamma} f(t)=\frac{(-1)^{n}}{\Gamma(n-\gamma)} \frac{d^{n}}{d t^{n}} \int_{t}^{b}(s-t)^{n-\gamma-1} f(s) d s, \quad t \in[a, b]
$$

where $n-1 \leq \gamma<n$ and $n \in \mathbb{N}$. In particular, if $0 \leq \gamma<1$, then

$$
{ }_{a} D_{t}^{\gamma} f(t)=\frac{1}{\Gamma(1-\gamma)} \frac{d}{d t} \int_{a}^{t}(t-s)^{-\gamma} f(s) d s, \quad t \in[a, b],
$$

and

$$
{ }_{t} D_{b}^{\gamma} f(t)=-\frac{1}{\Gamma(1-\gamma)} \frac{d}{d t} \int_{t}^{b}(s-t)^{-\gamma} f(s) d s, \quad t \in[a, b] .
$$

Definition 2.3 ([6]) If $\gamma \in(n-1, n)$ and $f \in A C^{n}([a, b], \mathbb{R})$, then the left and right Caputo fractional derivatives of order $\gamma$ for function $f$ denoted by ${ }_{a}^{c} D_{t}^{\gamma} f(t)$ and ${ }_{t}^{c} D_{b}^{\gamma} f(t)$, respectively, exist almost everywhere on $[a, b] .{ }_{a}^{c} D_{t}^{\gamma} f(t)$ and ${ }_{t}^{c} D_{b}^{\gamma} f(t)$ are represented by

$$
{ }_{a}^{c} D_{t}^{\gamma} f(t)={ }_{a} D_{t}^{\gamma-n} f^{(n)}(t)=\frac{1}{\Gamma(n-\gamma)} \int_{a}^{t}(t-s)^{n-\gamma-1} f^{(n)}(s) d s
$$

and

$$
{ }_{t}^{c} D_{b}^{\gamma} f(t)=(-1)^{n}{ }_{t} D_{b}^{\gamma-n} f^{(n)}(t)=\frac{(-1)^{n}}{\Gamma(n-\gamma)} \int_{t}^{b}(s-t)^{n-\gamma-1} f^{(n)}(s) d s,
$$

respectively, where $t \in[a, b]$. In particular, if $0<\gamma<1$, then

$$
{ }_{a}^{c} D_{t}^{\gamma} f(t)={ }_{a} D_{t}^{\gamma-1} f^{\prime}(t)=\frac{1}{\Gamma(1-\gamma)} \int_{a}^{t}(t-s)^{-\gamma} f^{\prime}(s) d s, \quad t \in[a, b]
$$

and

$$
{ }_{t}^{c} D_{b}^{\gamma} f(t)=-{ }_{t} D_{b}^{\gamma-1} f^{\prime}(t)=-\frac{1}{\Gamma(1-\gamma)} \int_{t}^{b}(s-t)^{-\gamma} f^{\prime}(s) d s, \quad t \in[a, b] .
$$


Let us recall that, for any $u \in L^{p}[0, T], 1 \leq p<\infty,\|u\|_{p}=\left(\int_{0}^{T}|u(t)|^{p} d t\right)^{1 / p}$, and $u \in$ $C[0, T],\|u\|_{\infty}=\max _{t \in[0, T]}|u(t)|$.

Definition 2.4 Let $0<\alpha \leq 1$ and $1<p<\infty$. The fractional derivative space $E_{0}^{\alpha, p}$ is defined by the closure of $C_{0}^{\infty}([0, T], \mathbb{R})$ with respect to the weighted norm

$$
\|u\|_{\alpha, p}=\left(\int_{0}^{T}|u(t)|^{p} d t+\int_{0}^{T}\left|{ }_{0}^{c} D_{t}^{\alpha} u(t)\right|^{p} d t\right)^{1 / p}, \quad \forall u \in E_{0}^{\alpha, p} .
$$

As in [7], we note the following.

\section{Remark 2.1}

(1) The fractional derivative space $E_{0}^{\alpha, p}$ is the space of functions $u \in L^{p}([0, T], \mathbb{R})$ having an $\alpha$-order Caputo fractional derivative ${ }_{0}^{c} D_{t}^{\alpha} u \in L^{p}([0, T], \mathbb{R})$ and $u(0)=u(T)=0$.

(2) For any $u \in E_{0}^{\alpha, p}$, noting the fact that $u(0)=0$, we have ${ }_{0}^{c} D_{t}^{\alpha} u(t)={ }_{0} D_{t}^{\alpha} u(t), t \in[0, T]$.

Lemma 2.1 ([7]) Let $0<\alpha \leq 1$ and $1<p<\infty$. The fractional derivative space $E_{0}^{\alpha, p}$ is a reflexive and separable Banach space.

Lemma 2.2 ([7]) Let $0<\alpha \leq 1$ and $1<p<\infty$. For any $u \in E_{0}^{\alpha, p}$, we have

$$
\|u\|_{p} \leq \frac{T^{\alpha}}{\Gamma(\alpha+1)}\left\|{ }_{0}^{c} D_{t}^{\alpha} u\right\|_{p}
$$

Moreover, if $\alpha>1 / p$ and $1 / p+1 / q=1$, then

$$
\|u\|_{\infty} \leq \frac{T^{\alpha-1 / p}}{\Gamma(\alpha)((\alpha-1) q+1)^{1 / q}}\left\|{ }_{0}^{c} D_{t}^{\alpha} u\right\|_{p}
$$

According to Lemma 2.2, we can also consider the space $E_{0}^{\alpha, p}$ with respect to the equivalent norm,

$$
\|u\|_{\alpha, p}=\left\|{ }_{0}^{c} D_{t}^{\alpha} u\right\|_{L^{p}}=\left(\int_{0}^{T}\left|{ }_{0}^{c} D_{t}^{\alpha} u(t)\right|^{p} d t\right)^{\frac{1}{p}}, \quad \forall u \in E_{0}^{\alpha, p} .
$$

Lemma 2.3 ([7]) Let $0<\alpha \leq 1$ and $1<p<\infty$. If $\alpha>1 / p$ and the sequence $\left\{u_{n}\right\}$ converges weakly to u in $E_{0}^{\alpha, p} ;$ i.e., $u_{n} \rightarrow u$, then $u_{n} \rightarrow$ u in $C([0, T], \mathbb{R})$, i.e., $\left\|u_{n}-u\right\|_{\infty} \rightarrow 0$, as $n \rightarrow \infty$.

In this paper, we consider problem (1.2) in the context of the Hilbert space $X^{\alpha}:=E_{0}^{\alpha, 2}$ furnished with the norm $\|u\|_{\alpha}=\|u\|_{\alpha, 2}$ as defined in (2.1). Note that, under certain conditions imposed on the function $a$, we also consider the inner product

$$
\begin{gathered}
(u, v):=\int_{0}^{T}\left(\left({ }_{0}^{c} D_{t}^{\alpha} u(t)\right)\left({ }_{0}^{c} D_{t}^{\alpha} v(t)\right)+a(t) u(t) v(t)\right) d t, \\
\forall u, v \in X^{\alpha}, \quad \text { which induces the norm } \\
\|u\|:=\left(\int_{0}^{T}\left(\left|{ }_{0}^{c} D_{t}^{\alpha} u(t)\right|^{2}+a(t)|u(t)|^{2}\right) d t\right)^{\frac{1}{2}}
\end{gathered}
$$

this is equivalent to $\|u\|_{\alpha, 2}$ as defined in (2.1). 
Definition 2.5 A function $u \in\left\{u \in A C([0, T]): \int_{t_{j}}^{t_{j+1}}\left(\left.\left.\right|_{0} ^{c} D_{t}^{\alpha} u(t)\right|^{2}+|u(t)|^{2}\right) d t<\infty, j=\right.$ $0,1,2, \ldots, n\}$ is said to be a classical solution of problem (1.2), if $u$ satisfies the equation a.e. on $[0, T] \backslash\left\{t_{1}, t_{2}, \ldots, t_{n}\right\}$, the limits ${ }_{t} D_{T}^{\alpha-1}\left({ }_{0}^{c} D_{t}^{\alpha} u\right)\left(t_{j}^{+}\right)$, and the limits $D_{T}^{\alpha-1}\left({ }_{0}^{c} D_{t}^{\alpha} u\right)\left(t_{j}^{-}\right)$exist and satisfy the impulsive conditions

$$
\Delta\left({ }_{t} D_{T}^{\alpha-1}\left({ }_{0}^{c} D_{t}^{\alpha} u\right)\right)\left(t_{j}\right)=I_{j}\left(u\left(t_{j}\right)\right), \quad j=1,2, \ldots, n,
$$

and the boundary condition $u(0)=u(T)=0$ holds.

Definition 2.6 A function $u \in X^{\alpha}$ is said to be a weak solution of problem (1.2) if, for every $v \in X^{\alpha}$, the following identity holds:

$$
\begin{aligned}
& \int_{0}^{T}\left(\left({ }_{0}^{c} D_{t}^{\alpha} u(t)\right)\left({ }_{0}^{c} D_{t}^{\alpha} v(t)\right)+a(t) u(t) v(t)\right) d t+\sum_{j=1}^{n} I_{j}\left(u\left(t_{j}\right)\right) v(t) \\
& =\int_{0}^{T} f\left(t, u(t),{ }_{0}^{c} D_{t}^{\alpha} u(t)\right) v(t) d t .
\end{aligned}
$$

By a discussion similar to [16], we can obtain the following lemma.

Lemma 2.4 The function $u \in X^{\alpha}$ is a weak solution of (1.2) if and only if $u$ is a classical solution of (1.2).

For the following $B V P$ :

$$
\left\{\begin{array}{l}
{ }_{t} D_{T}^{\alpha}\left({ }_{0}^{c} D_{t}^{\alpha} u(t)\right)=\lambda u(t), \quad t \in[0, T] \\
u(0)=u(T)=0
\end{array}\right.
$$

in terms of [18], we call $u \in X^{\alpha} \backslash\{0\}$ is a eigenvector with respect to the eigenvalue $\lambda$, if $u \in X^{\alpha}$ satisfies BVP (2.3). Similarly, by [18], we call $u \in X^{\alpha}$ is a weak solution (2.3) if

$$
\int_{0}^{T}\left({ }_{0}^{c} D_{t}^{\alpha} u(t)\right)\left({ }_{0}^{c} D_{t}^{\alpha} \nu(t)\right) d t=\lambda \int_{0}^{T} u(t) v(t) d t
$$

holds for every $v \in X^{\alpha}$. Certainly, $u$ is a classical solution of $B V P$ (2.3) if only if $u \in X^{\alpha}$ is a weak solution of $B V P(2.3)$.

The following two lemmas are established in [18].

Lemma 2.5 Suppose that $0<\alpha \leq 1$. Then each eigenvalue of problem (2.3) is real and, if we repeat each eigenvalue according to its multiplicity, we have $0<\lambda_{1} \leq \lambda_{2} \leq \lambda_{3} \leq \cdots$ and $\lambda_{k} \rightarrow \infty$ as $k \rightarrow \infty$. In particular, $\lambda_{1}$ can be characterized as

$$
\lambda_{1}=\inf _{u \in X^{\alpha} \backslash\{0\}} \frac{\left.\left.\int_{0}^{T}\right|_{0} ^{c} D_{t}^{\alpha} u(t)\right|^{2} d t}{\int_{0}^{T}|u(t)|^{2} d t} .
$$

Lemma 2.6 Suppose that $0<\alpha \leq 1$. If $\operatorname{essinf}_{t \in[0, T]} a(t)=m>-\lambda_{1}$, then the norm $\|\cdot\|$ and the norm $\|\cdot\|_{\alpha, 2}$ are equivalent, i.e., there exist two positive constants $\eta_{1}, \eta_{2}$ such that

$$
\eta_{1}\|u\|_{\alpha, 2} \leq\|u\| \leq \eta_{2}\|u\|_{\alpha, 2}, \quad \forall u \in X^{\alpha} .
$$


As in the proof of Lemma 5 [18], we can take $\eta_{1}=\sqrt{\varepsilon}$ and $\eta_{2}=\left(1+\frac{\|a\|_{\infty}}{\lambda_{1}}\right)^{\frac{1}{2}}$. We take $\varepsilon=$ $\min \left\{\frac{3}{4}, \frac{\lambda_{1}+m}{\lambda_{1}}\right\}$.

To establish our result, we consider the function $\phi_{w}: X^{\alpha} \rightarrow \mathbb{R}$ for any fixed $w \in X^{\alpha}$ as follows:

$$
\begin{aligned}
\phi_{w}(u)= & \frac{1}{2} \int_{0}^{T}\left(\left|{ }_{0}^{c} D_{t}^{\alpha} u(t)\right|^{2}+a(t)|u(t)|^{2}\right) d t+\sum_{j=1}^{n} \int_{0}^{u\left(t_{j}\right)} I_{j}(s) d s \\
& -\int_{0}^{T} F\left(t, u(t),{ }_{0}^{c} D_{t}^{\alpha} w(t)\right) d t
\end{aligned}
$$

for $u \in X^{\alpha}$, where $F(t, u, y)=\int_{0}^{u} f(t, s, y) d s$.

Similarly to [18], by the continuity of $a, f$ and $I_{j}$, the functional $\phi_{w}$ is clearly continuous and differentiable on $X^{\alpha}$ and for every $u, v \in X^{\alpha}$, the following relation holds:

$$
\begin{aligned}
\phi_{w}^{\prime}(u) v= & \int_{0}^{T}\left[\left({ }_{0}^{c} D_{t}^{\alpha} u(t)\right)\left({ }_{0}^{c} D_{t}^{\alpha} v(t)\right)+a(t) u(t) v(t)\right] d t \\
& +\sum_{j=1}^{n} I_{j}\left(u\left(t_{j}\right)\right) v\left(t_{j}\right)-\int_{0}^{T} f\left(t, u(t),{ }_{0}^{c} D_{t}^{\alpha} w(t)\right) v(t) d t .
\end{aligned}
$$

Hence, $u \in X^{\alpha}$ is a weak solution of BVP (1.2) if and only if $u \in X^{\alpha}$ satisfies $\phi_{u}^{\prime}(u) v=0$ for all $v \in X^{\alpha}$.

For convenience, we state some necessary definitions and theorem.

Definition 2.7 ([24]) Suppose that $X$ is a Banach space and $\phi \in C^{1}(X, \mathbb{R})$. We say that $\phi$ satisfies the Palais-Smale condition if any sequence $\left\{u_{n}\right\} \subset X$ such that $\phi\left(u_{n}\right)$ is bounded and $\phi_{n}^{\prime}\left(u_{n}\right) \rightarrow 0$ as $n \rightarrow \infty$ possesses a convergent subsequence in $X$.

Lemma 2.7 ([24]; mountain pass theorem) Let $X$ be a Banach space and let $\phi \in C^{1}(X, \mathbb{R})$ satisfy the Palais-Smale condition. Assume that there exist $u_{0}, u_{1} \in X$ and a bounded open neighborhood $\Omega$ of $u_{0}$ such that $u_{1} \in X \backslash \bar{\Omega}$ and $\max \left\{\phi\left(u_{0}\right), \phi\left(u_{1}\right)\right\}<\inf _{v \in \partial \Omega} \phi(v)$. Let

$$
\Lambda=\left\{h \in C([0,1], X): h(0)=u_{0}, h(1)=u_{1}\right\}, \quad \tau=\inf _{h \in \Lambda, s \in[0,1]} \phi(h(s)) .
$$

Then $\tau$ is a critical value of $\phi$, that is, there exists $u^{*}$ such that $\phi^{\prime}\left(u^{*}\right)=0$ and $\phi\left(u^{*}\right)=\tau$, where $\tau>\max \left\{\phi\left(u_{0}\right), \phi\left(u_{1}\right)\right\}$.

Definition 2.8 ([25]) We say that $\phi$ satisfies condition (C) if, for any $\left\{u_{n}\right\} \subset X,\left\{u_{n}\right\}$ has a convergent subsequence if $\phi\left(u_{n}\right)$ is bounded and $\left(1+\left\|u_{n}\right\|\right)\left\|\phi^{\prime}\left(u_{n}\right)\right\| \rightarrow 0$ as $n \rightarrow \infty$.

As shown in [25], a deformation lemma can be proved with condition $(C)$ replacing the Palais-Smale condition and it turns out that the mountain pass theorem holds true under condition (C).

\section{Main result}

For convenience, we first list the following conditions which will be used in this paper. 
$\left(H_{1}\right)$ There exist constants $b \geq 0, c \geq 0, \delta>0, b_{j} \geq 0, j=1,2, \ldots, n$, and $\gamma>1, \xi>1,0<\theta<$ $2, \gamma_{j}>1, j=1,2, \ldots, n$, such that

$$
\begin{aligned}
& f(t, x, y) \leq b|x|^{\gamma}+c|x|^{\xi}|y|^{\theta}, \quad \text { for }|x| \leq \delta, y \in \mathbb{R} \text {, a.e. } t \in[0, T], \\
& I_{j}(s) \geq-b_{j}|s|^{\gamma_{j}}, \quad j=1,2, \ldots, n, \text { for }|s| \leq \delta .
\end{aligned}
$$

$\left(H_{2}\right)$ There exist constants $\mu>2, l \geq 0, m \geq 0, d \geq 0,0<\sigma, \tau, \sigma_{j}<2, l_{j} \geq 0, j=1,2, \ldots, n$, and $L \geq 0$, such that

$$
\begin{aligned}
& x f(t, x, y)-\mu F(t, x, y) \geq-l|x|^{\sigma}-m|y|^{\tau}-d, \quad \text { for } x, y \in \mathbb{R} \text {, a.e. } t \in[0, T], \\
& \mu \int_{0}^{u} I_{j}(s) d s-I_{j}(u) u \geq-l_{j}|u|^{\sigma_{j}}, \quad \text { for }|u| \geq L .
\end{aligned}
$$

$\left(H_{3}\right)$ There exist constants $\beta>0, \lambda \geq 0, J \geq 0, M \geq 0, \beta_{j} \geq 0, j=1,2, \ldots, n$, and $\omega>1$, $0<\zeta<1,0<\omega_{j}<1, j=1,2, \ldots, n$, such that

$$
\begin{aligned}
& f(t, x, y) \geq \beta x^{\omega}-\lambda|y|^{\zeta}-M, \quad x \geq 0, y \in \mathbb{R} \text {, a.e. } t \in[0, T], \\
& I_{j}(s) \leq \beta_{j} \omega^{\omega_{j}}, \quad s \geq J .
\end{aligned}
$$

$\left(H_{4}\right)$ There exist nonnegative functions $p, q \in L^{2}$, and constants $a_{j}>0, j=1,2, \ldots, n$, such that

$$
\left|f\left(t, x_{2}, y_{2}\right)-f\left(t, x_{1}, y_{1}\right)\right| \leq p(t)\left|x_{2}-x_{1}\right|+q(t)\left|y_{2}-y_{1}\right|
$$

for $x_{1}, x_{2} \in\left[-K_{1}, K_{1}\right]$ and $y_{1}, y_{2} \in \mathbb{R}$, a.e. $t \in[0, T]$,

$$
\left|I_{j}(x)-I_{j}(y)\right| \leq a_{j}|x-y|
$$

for all $x, y \in\left[-K_{1}, K_{1}\right]$, where $K_{1}=\frac{T^{\alpha-1 / 2}}{\eta_{1} \Gamma(\alpha) \sqrt{2 \alpha-1}} K$ and $K$ is described as in the sequel.

We give some notations which will be used in the sequel:

$$
\begin{aligned}
& \bar{u}_{0}=\frac{1}{\Lambda_{0}} u_{0} \in X^{\alpha}, \quad \Lambda_{0}^{2}=\left\|u_{0}\right\|^{2}=\int_{0}^{T}\left(\left|{ }_{0}^{c} D_{t}^{\alpha} u_{0}(t)\right|^{2}+a(t) u_{0}^{2}(t)\right) d t \quad \text { and } \\
& u_{0}= \begin{cases}\frac{4}{T} t, & t \in[0, T / 4], \\
1, & t \in[T / 4,3 T / 4], \quad \bar{\beta}=\frac{\beta T(\omega+3)}{2(\omega+1)(\omega+2) \Lambda_{0}^{\omega+1}}, \quad \bar{M}=\frac{3 M T}{4 \Lambda_{0}}, \\
\frac{4}{T}(T-t), & t \in(3 T / 4, T],\end{cases} \\
& \bar{\beta}_{j}=\frac{\beta_{j}\left(\bar{u}_{0}\left(t_{j}\right)\right)^{\omega_{j}+1}}{\omega_{j}+1}, \quad \bar{M}_{j}=M_{j} \bar{u}_{0}\left(t_{j}\right), \quad \bar{d}=\sum_{j=1}^{n} d_{j}+d T, \quad M_{j}=\max _{0 \leq s \leq J}\left|I_{j}(s)\right|, \\
& d_{j}=\max _{|x| \leq L}\left|\mu \int_{0}^{x} I_{j}(s) d s-I_{j}(x) x\right|, \quad \bar{m}=T^{\frac{2-\tau}{\tau}} \eta_{1}^{-\tau} m, \quad \bar{l}=l T^{\frac{2-\sigma}{\sigma}}\left(\frac{T^{\alpha}}{\eta_{1} \Gamma(\alpha+1)}\right)^{\sigma}, \\
& \bar{l}_{j}=l_{j}\left(\frac{T^{\alpha-1 / 2}}{\eta_{1} \Gamma(\alpha) \sqrt{2 \alpha-1}}\right)^{\sigma_{j}}, \quad \bar{\lambda}=\frac{\lambda}{\eta_{1}^{\zeta} \Lambda_{0}}\left(\frac{T(3-\zeta)}{4-\zeta}\right)^{\frac{2-\zeta}{2}},
\end{aligned}
$$




$$
\begin{aligned}
& \bar{r}=\frac{2-\zeta}{2} \bar{\lambda}^{\frac{2}{2-\zeta}}\left(\frac{8 \mu \zeta}{\mu-2}\right)^{\frac{\zeta}{\mu-2}}, \quad m^{*}=\frac{2-\tau}{2} \bar{m}^{\frac{2}{2-\tau}}\left(\frac{8 \tau}{\mu-2}\right)^{\frac{\tau}{2-\tau}}, \\
& l^{*}=\frac{2-\sigma}{2} \bar{l}^{\frac{2}{2-\sigma}}\left(\frac{8 \sigma}{\mu-2}\right)^{\frac{\sigma}{2-\sigma}}, \quad l_{j}^{*}=\frac{2-\sigma_{j}}{2} \bar{l}_{j}^{2-\sigma_{j}}\left(\frac{8 n \sigma_{j}}{\mu-2}\right)^{\frac{\sigma_{j}}{2-\sigma_{j}}}, \\
& A=\frac{1}{2}+\sum_{j=1}^{n} \bar{\beta}_{j}+\sum_{j=1}^{n} \bar{M}_{j}+\bar{M}+\bar{r}, \quad B=\frac{(\omega-1) A}{\omega+1}\left(\frac{2 A}{\bar{\beta}(\omega+1)}\right)^{\frac{2}{\omega-1}}, \\
& C=\max \{A, B\}, \quad E=\frac{2}{\mu-2}\left(\mu C+m^{*}+l^{*}+\sum_{j=1}^{n} l_{j}^{*}+\bar{d}\right), \quad K=\sqrt{2 E}, \\
& P_{0}=\frac{T^{2 \alpha-1 / 2}}{\Gamma(\alpha) \Gamma(\alpha+1) \sqrt{2 \alpha-1} \eta_{1}^{2}}\|p\|_{2}+\frac{n}{(\Gamma(\alpha))^{2}(2 \alpha-1) \eta_{1}^{2}} \sum_{j=1}^{n} a_{j}, \\
& Q_{0}=\frac{T^{\alpha-1 / 2}}{\Gamma(\alpha) \sqrt{2 \alpha-1} \eta_{1}^{2}}\|q\|_{2} .
\end{aligned}
$$

The above $\beta, \beta_{j}, d, d_{j}, M, M_{j}, \ldots$ etc., are described as in $\left(H_{1}\right)-\left(H_{4}\right)$.

We are in a position to state our result as below.

Theorem 3.1 Suppose that $\frac{1}{2}<\alpha \leq 1$, $\inf _{t \in[0, T]} a(t)=m>-\lambda_{1}$ and the conditions $\left(H_{1}\right)$ $\left(H_{4}\right)$ hold. Moreover, assume that $P_{0}<1$ and $\frac{Q_{0}}{1-P_{0}}<1$. Then problem (1.2) has a nontrivial classical solution.

Proof The proof is divided into five steps.

Step 1. For any fixed $w \in X^{\alpha}$ with $\|w\| \leq K$. Take $\delta_{1}=\frac{\sqrt{2 \alpha-1} \Gamma(\alpha) \eta_{1} \delta}{T^{\alpha-1 / 2}}$, then, for any $u \in X^{\alpha}$ with $\|u\| \leq \delta_{1}$, it follows from Lemma 2.2 and Lemma 2.6 that $|u(t)| \leq \delta$ for all $t \in[0, T]$. Thus, by $\left(H_{1}\right)$ we have

$$
\begin{aligned}
& f\left(t, s,{ }_{0}^{c} D_{t}^{\alpha} w(t)\right) \leq b|s|^{\gamma}+c|s|^{\xi}\left|{ }_{0}^{c} D_{t}^{\alpha} w(t)\right|^{\theta}, \quad \text { for a.e. } t \in[0, T],|s| \leq|u(t)| \\
& I_{j}(s) \geq-b_{j}|s|^{\gamma_{j}}, \quad|s| \leq\left|u\left(t_{j}\right)\right|, \quad j=1,2, \ldots, n,
\end{aligned}
$$

and therefore

$$
\begin{aligned}
F\left(t, u(t),{ }_{0}^{c} D_{t}^{\alpha} w(t)\right) & =\int_{0}^{u(t)} f\left(t, s,{ }_{0}^{c} D_{t}^{\alpha} w(t)\right) d s \\
& \leq \int_{0}^{|u(t)|}\left(b|s|^{\gamma}+c|s|^{\xi}\left|{ }_{0}^{c} D_{t}^{\alpha} w(t)\right|^{\theta}\right) d s \\
& =\frac{b}{\gamma+1}|u(t)|^{\gamma+1}+\frac{c}{\xi+1}|u(t)|^{\xi+1}\left|{ }_{0}^{c} D_{t}^{\alpha} w(t)\right|^{\theta}, \quad \text { for a.e. } t \in[0, T] .
\end{aligned}
$$

Thus, in view of Lemma 2.2 and Lemma 2.6, we have

$$
\begin{aligned}
& \int_{0}^{T} F\left(t, u(t),{ }_{0}^{c} D_{t}^{\alpha} w(t)\right) d t \\
& \quad \leq \frac{b T}{\gamma+1}\|u\|_{\infty}^{\gamma+1}+\frac{c}{\xi+1}\|u\|_{\infty}^{\xi+1} \int_{0}^{T}\left|{ }_{0}^{c} D_{t}^{\alpha} w(t)\right|^{\theta} d t
\end{aligned}
$$




$$
\begin{aligned}
\leq & \frac{b T}{\gamma+1}\left(\frac{T^{\alpha-1 / 2}}{\eta_{1} \Gamma(\alpha) \sqrt{2 \alpha-1}}\right)^{\gamma+1}\|u\|^{\gamma+1} \\
& +\frac{c}{(\xi+1) \eta_{1}^{\theta}}\left(\frac{T^{\alpha-1 / 2}}{\eta_{1} \Gamma(\alpha) \sqrt{2 \alpha-1}}\right)^{\xi+1} T^{\frac{2-\theta}{2}}\|w\|^{\theta}\|u\|^{\xi+1} \\
= & \bar{b}\|u\|^{\gamma+1}+\bar{c}\|w\|^{\theta}\|u\|^{\xi+1} \leq \bar{b}\|u\|^{\gamma+1}+\bar{c} K^{\theta}\|u\|^{\xi+1}
\end{aligned}
$$

where $\bar{b}=\frac{b T}{\gamma+1}\left(\frac{T^{\alpha-1 / 2}}{\eta_{1} \Gamma(\alpha) \sqrt{2 \alpha-1}}\right)^{\gamma+1}, \bar{c}=\frac{c}{(\xi+1) \eta_{1}^{\theta}}\left(\frac{T^{\alpha-1 / 2}}{\eta_{1} \Gamma(\alpha) \sqrt{2 \alpha-1}}\right)^{\xi+1} T^{\frac{2-\theta}{2}}$ and

$$
\begin{aligned}
\int_{0}^{u\left(t_{j}\right)} I_{j}(s) d s & \geq-b_{j} \int_{0}^{\left|u\left(t_{j}\right)\right|}|s|^{\gamma_{j}} d s=-\frac{b_{j}}{\gamma_{j}+1}\left|u\left(t_{j}\right)\right|^{\gamma_{j}+1} \geq-\frac{b_{j}}{\gamma_{j}+1}\|u\|_{\infty}^{\gamma_{j}+1} \\
& \geq-\frac{b_{j}}{\gamma_{j}+1}\left(\frac{T^{\alpha-1 / 2}}{\eta_{1} \Gamma(\alpha) \sqrt{2 \alpha-1}}\right)^{\gamma_{j}+1}\|u\|^{\gamma_{j}+1}=-\overline{b_{j}}\|u\|^{\gamma_{j}+1},
\end{aligned}
$$

where $\overline{b_{j}}=\frac{b_{j}}{\gamma_{j}+1}\left(\frac{T^{\alpha-1 / 2}}{\eta_{1} \Gamma(\alpha) \sqrt{2 \alpha-1}}\right)^{\gamma_{j}+1}$. Hence, by (2.4), we have

$$
\phi_{w}(u) \geq \frac{1}{2}\|u\|^{2}-\sum_{j=1}^{n} \overline{b_{j}}\|u\|^{\gamma_{j}+1}-\bar{b}\|u\|^{\gamma+1}-\bar{c} K^{\theta}\|u\|^{\xi+1} .
$$

Owing to the fact that $\gamma>1, \gamma_{j}>1, j=1,2, \ldots, n$ and $\xi>1$, we can choose $\rho$ small enough so that

$$
\frac{1}{2}-\sum_{j=1}^{n} \overline{b_{j}} \rho^{\gamma_{j}-1}-\bar{b} \rho^{\gamma-1}-\bar{c} K^{\theta} \rho^{\xi-1}>\frac{1}{4} .
$$

Then

$$
\phi_{w}(u) \geq \frac{1}{4} \rho^{2}:=\alpha_{1}>0
$$

for any $u \in X^{\alpha}$ with $\|u\|=\rho$.

Step 2. We show that $\phi_{w}$ satisfies (C) condition, i.e., for any $\left\{u_{n}\right\} \subset X^{\alpha}$ has a convergent subsequence if $\left\{\phi_{w}\left(u_{n}\right)\right\}$ is bounded and $\left(1+\left\|u_{n}\right\|\right)\left\|\phi_{w}^{\prime}\left(u_{n}\right)\right\| \rightarrow 0$ as $n \rightarrow \infty$.

Let

$$
d_{j}=\max _{|x| \leq L}\left|\mu \int_{0}^{x} I_{j}(s) d s-I_{j}(x) x\right|, \quad j=1,2, \ldots, n .
$$

Then by $\left(H_{2}\right)$, we have

$$
\begin{aligned}
& x f(t, x, y)-\mu F(t, x, y) \geq-l|x|^{\sigma}-m|y|^{\tau}-d, \quad \text { for } x, y \in \mathbb{R}, \text { a.e. } t \in[0, T], \\
& \mu \int_{0}^{u} I_{j}(s) d s-I_{j}(u) u \geq-l_{j}|u|^{\sigma_{j}}-d_{j}, \quad j=1,2, \ldots, n, u \in \mathbb{R} .
\end{aligned}
$$

Thus, by Lemma 2.2 and Lemma 2.6, it follows from (2.4)-(2.5) that

$$
\begin{aligned}
& \mu \phi_{w}\left(u_{k}\right)-\phi_{w}^{\prime}\left(u_{k}\right) u_{k} \\
& \quad=\left(\frac{\mu}{2}-1\right)\left\|u_{k}\right\|^{2}+\int_{0}^{T}\left(f\left(t, u_{k}(t),{ }_{0}^{c} D_{t}^{\alpha} w(t)\right) u_{k}(t)-\mu F\left(t, u_{k}(t),{ }_{0}^{c} D_{t}^{\alpha} w(t)\right)\right) d t
\end{aligned}
$$




$$
\begin{aligned}
& +\sum_{j=1}^{n}\left(\mu \int_{0}^{u_{k}\left(t_{j}\right)} I_{j}(s) d s-I_{j}\left(u_{k}\left(t_{j}\right)\right) u_{k}\left(t_{j}\right)\right) \\
\geq & \left(\frac{\mu}{2}-1\right)\left\|u_{k}\right\|^{2}-\left(l \int_{0}^{T}\left(\left|u_{k}(t)\right|^{\sigma} d t+m \int_{0}^{T}\left|{ }_{0}^{c} D_{t}^{\alpha} w(t)\right|^{\tau} d t\right)\right. \\
& -\left(\sum_{j=1}^{n}\left(l_{j}\left|u_{k}\left(t_{j}\right)\right|^{\sigma_{j}}+d_{j}\right)+d T\right) \\
\geq & \left(\frac{\mu}{2}-1\right)\left\|u_{k}\right\|^{2}-\left(T^{\frac{2-\sigma}{\sigma}} l\left\|u_{k}\right\|_{L^{2}}^{\sigma}+m \int_{0}^{T}\left|{ }_{0}^{c} D_{t}^{\alpha} w(t)\right|^{\tau} d t\right) \\
& -\left(\sum_{j=1}^{n} l_{j}\left\|u_{k}\right\|_{\infty}^{\sigma_{j}}+\sum_{j=1}^{n} d_{j}+d T\right) \\
\geq & \left(\frac{\mu}{2}-1\right)\left\|u_{k}\right\|^{2}-\left(l T^{\frac{2-\sigma}{\sigma}}\left(\frac{T^{\alpha}}{\eta_{1} \Gamma(\alpha+1)}\right)^{\sigma}\left\|u_{k}\right\|^{\sigma}+m \int_{0}^{T}\left|{ }_{0}^{c} D_{t}^{\alpha} w(t)\right|^{\tau} d t\right) \\
& -\left(\sum_{j=1}^{n} l_{j}\left(\frac{T^{\alpha-1 / 2}}{\eta_{1} \Gamma(\alpha) \sqrt{2 \alpha-1}}\right)^{\sigma_{j}}\left\|u_{k}\right\|^{\sigma_{j}}+\sum_{j=1}^{n} d_{j}+d T\right) \\
= & \left(\frac{\mu}{2}-1\right)\left\|u_{k}\right\|^{2}-\left(\bar{l}\left\|u_{k}\right\|^{\sigma}+m \int_{0}^{T}\left|{ }_{0}^{c} D_{t}^{\alpha} w(t)\right|^{\tau} d t\right) \\
& -\left(\sum_{j=1}^{n} \bar{l}_{j}\left\|u_{k}\right\|^{\sigma_{j}}+\sum_{j=1}^{n} d_{j}+d T\right),
\end{aligned}
$$

where $\bar{l}=l T^{\frac{2-\sigma}{\sigma}}\left(\frac{T^{\alpha}}{\eta_{1} \Gamma(\alpha+1)}\right)^{\sigma}, \bar{l}_{j}=l_{j}\left(\frac{T^{\alpha-1 / 2}}{\eta_{1} \Gamma(\alpha) \sqrt{2 \alpha-1}}\right)^{\sigma_{j}}$.

Hence, noting that the assumption $0<\sigma, \sigma_{j}<2, \mu>2$ and the fact that $\left\{\phi_{w}\left(u_{k}\right)\right\}$ is bounded and $\phi_{w}^{\prime}\left(u_{k}\right) u_{k} \rightarrow 0$ as $k \rightarrow \infty$, from inequality (3.2), we see that $\left\{u_{k}\right\}$ is bounded in $X^{\alpha}$. So, in view of the reflexivity of space, there exists a subsequence $\left\{u_{k_{j}}\right\}$ with $u_{k_{j}} \rightarrow$ $u \in X^{\alpha}$. For simplicity, we still denote $\left\{u_{k_{j}}\right\}$ by $\left\{u_{k}\right\}$. It follows from Lemma 2.2 that $u_{k} \rightarrow u$ in $C[0, T]$. Owing to the fact that $\phi_{w}^{\prime}\left(u_{k}\right) \rightarrow 0, u_{k} \rightarrow u$ as $k \rightarrow \infty$, and boundedness of the sequence $\left\{u_{k}-u\right\}$, we get

$$
\left|\left(\phi_{w}^{\prime}\left(u_{k}\right)-\phi_{w}^{\prime}(u)\right)\left(u_{k}-u\right)\right| \leq\left|\phi_{w}^{\prime}\left(u_{k}\right)\right|\left\|u_{k}-u\right\|+\left|\phi_{w}^{\prime}(u)\left(u_{k}-u\right)\right| \rightarrow 0
$$

as $k \rightarrow \infty$. Thus

$$
\begin{aligned}
\left\|u_{k}-u\right\|^{2}= & \left(\phi_{w}^{\prime}\left(u_{k}\right)-\phi_{w}^{\prime}(u)\right)\left(u_{k}-u\right)-\sum_{j=1}^{n}\left[I_{j}\left(u_{k}\left(t_{j}\right)\right)-I_{j}\left(u\left(t_{j}\right)\right)\right]\left(u_{k}\left(t_{j}\right)-u\left(t_{j}\right)\right) \\
& +\int_{0}^{T}\left(f\left(t, u_{k}(t),{ }_{0}^{c} D_{t}^{\alpha} w(t)\right)-f\left(t, u(t),{ }_{0}^{c} D_{t}^{\alpha} w(t)\right)\right)\left(u_{k}(t)-u(t)\right) d t \rightarrow 0
\end{aligned}
$$

as $k \rightarrow \infty$, because of the continuity of $f$ and $I_{j}, j=1,2, \ldots, n$, and the fact that $u_{k} \rightarrow u$ in $C[0, T]$. Thus $\phi_{w}(u)$ satisfies the condition (C).

Step 3 . We will show that there exists a point $u^{*} \in X^{\alpha}$ with $\left\|u^{*}\right\|>\rho$ satisfying $\phi_{w}\left(u^{*}\right)<0$. In fact, let $M_{j}=\max _{0 \leq s \leq J}\left|I_{j}(s)\right|$, then by $\left(H_{3}\right)$ we have

$$
\begin{aligned}
& f(t, x, y) \geq \beta x^{\omega}-\lambda|y|^{\zeta}-M, \quad 0 \leq x<\infty, y \in \mathbb{R}, \text { a.e. } t \in[0, T] \\
& I_{j}(s) \leq \beta_{j} s^{\omega_{j}}+M_{j}, \quad 0 \leq s<\infty, j=1,2, \ldots, n,
\end{aligned}
$$


and therefore

$$
\begin{aligned}
& F(t, u, y)=\int_{0}^{u} f(t, s, y) d s \geq \frac{\beta}{\omega+1} u^{\omega+1}-\lambda u|y|^{\zeta}-M u, \quad \text { for } u \geq 0, \text { a.e. } t \in[0, T], \\
& \int_{0}^{u} I_{j}(s) d s \leq \frac{\beta_{j}}{\omega_{j}+1} u^{\omega_{j}+1}+M_{j} u, \quad \text { for any } u \geq 0 .
\end{aligned}
$$

As before, for the choice $\bar{u}_{0}=\frac{1}{\Lambda_{0}} u_{0} \in X^{\alpha}, \Lambda_{0}=\left\|u_{0}\right\|,\left\|\bar{u}_{0}\right\|=1$, and

$$
u_{0}= \begin{cases}\frac{4}{T} t, & t \in[0, T / 4] \\ 1, & t \in[T / 4,3 T / 4] \\ \frac{4}{T}(T-t), & t \in(3 T / 4, T]\end{cases}
$$

then $\bar{u}_{0}(t)>0, t \in(0, T), \Lambda_{0}^{2}=\int_{0}^{T}\left(\left.{ }_{0}^{c} D_{t}^{\alpha} u_{0}(t)\right|^{2}+a(t) u_{0}^{2}(t)\right) d t$, and

$$
{ }_{0}^{c} D_{t}^{\alpha} u_{0}(t)=\frac{4}{T \Gamma(2-\alpha)} \begin{cases}t^{1-\alpha}, & t \in[0, T / 4], \\ g(t), & t \in[T / 4,3 T / 4], \\ h(t), & t \in(3 T / 4, T],\end{cases}
$$

where $g(t)=t^{1-\alpha}-(t-T / 4)^{1-\alpha}, h(t)=t^{1-\alpha}-(t-T / 4)^{1-\alpha}-(t-3 T / 4)^{1-\alpha}$.

Hence, by (2.4), we have

$$
\begin{aligned}
\phi_{w}\left(\tau \bar{u}_{0}\right)= & \frac{1}{2} \tau^{2}+\sum_{j=1}^{n} \int_{0}^{\tau \bar{u}_{0}\left(t_{j}\right)} I_{j}(s) d s-\int_{0}^{T} F\left(t, \tau \bar{u}_{0}(t),{ }_{0}^{c} D_{t}^{\alpha} w(t)\right) d t \\
\leq & \frac{1}{2} \tau^{2}+\sum_{j=1}^{n}\left(\frac{\beta_{j}}{\omega_{j}+1} \tau^{\omega_{j}+1}\left(\bar{u}_{0}\left(t_{j}\right)\right)^{\omega_{j}+1}+\tau M_{j} \bar{u}_{0}\left(t_{j}\right)\right) \\
& -\int_{0}^{T}\left(\frac{\beta \tau^{\omega+1}}{\omega+1} \bar{u}_{0}(t)^{\omega+1}-\lambda \tau \bar{u}_{0}\left|{ }_{0}^{c} D_{t}^{\alpha} w(t)\right|^{\zeta}-M \tau \bar{u}_{0}\right) d t \\
= & \frac{1}{2} \tau^{2}+\sum_{j=1}^{n}\left(\tau^{\omega_{j}+1} \frac{\beta_{j}}{\omega_{j}+1}\left(\bar{u}_{0}\left(t_{j}\right)\right)^{\omega_{j}+1}+\tau M_{j} \bar{u}_{0}\left(t_{j}\right)\right) \\
& +\left.\left.\lambda \tau \int_{0}^{T} \bar{u}_{0}(t)\right|_{0} ^{c} D_{t}^{\alpha} w(t)\right|^{\zeta} d t \\
& +M \tau \int_{0}^{T} \bar{u}_{0}(t) d t-\frac{\beta}{\omega+1} \tau^{\omega+1} \int_{0}^{T}\left(\bar{u}_{0}(t)\right)^{\omega+1} d t
\end{aligned}
$$

for any $\tau>0$. Since $0<\omega_{j}<1, \omega>1$ and $\int_{0}^{T}\left(u_{0}(t)\right)^{\omega+1} d t>0$, from the above inequality, we see that there exists a $\tau_{0}>0$ large enough so that $\phi_{w}\left(\tau_{0} \bar{u}_{0}\right)<0$ with $\left\|\tau_{0} \bar{u}_{0}\right\|>\rho$. Let $u^{*}=\tau_{0} \bar{u}_{0}$, then $\phi_{w}\left(u^{*}\right)<0$ with $\left\|u^{*}\right\|>\rho$. Also, obviously, $\phi_{w}(0)=0$.

Now, applying Lemma 2.7 (the mountain pass theorem), we see that there exists a point $\bar{u} \in X^{\alpha}$ satisfying $\phi_{w}^{\prime}(\bar{u})=0$ and $\phi_{w}(\bar{u}) \geq \alpha_{1}>0$.

Step 4. We show that we can construct a sequence $\left\{u_{n}\right\}_{n=1}^{\infty}$ in $X^{\alpha}$ satisfying that $\phi_{u_{n-1}}^{\prime}\left(u_{n}\right)=0$ and $\phi_{u_{n-1}}\left(u_{n}\right) \geq \alpha_{1}$ with $\left\|u_{n}\right\| \leq K, n=1,2, \ldots$ 
First, for a certain $u_{1} \in X^{\alpha}$ with $\left\|u_{1}\right\| \leq K$, by the conclusion proved in Step 3, we know that there exists a point $u_{2} \in X^{\alpha}$ such that $\phi_{u_{1}}^{\prime}\left(u_{2}\right)=0$ with $\phi_{u_{1}}\left(u_{2}\right) \geq \alpha_{1}$. We claim that $\left\|u_{2}\right\| \leq K$.

In fact, by the previous proof in Step 3 and noting that we have (3.3), we obtain

$$
\begin{aligned}
\phi_{u_{1}}\left(u_{2}\right) \leq & \max _{\tau \in[0, \infty)} \phi_{u_{1}}\left(\tau \bar{u}_{0}\right) \\
\leq & \max _{\tau \in[0, \infty)}\left[\frac{1}{2} \tau^{2}+\sum_{j=1}^{n} \frac{\left(\bar{u}_{0}\left(t_{j}\right)\right)^{\omega_{j}+1} \beta_{j}}{\left(\omega_{j}+1\right)} \tau^{\omega_{j}+1}+\sum_{j=1}^{n} M_{j} \bar{u}_{0}\left(t_{j}\right) \tau\right. \\
& +\frac{\lambda}{\eta_{1}^{\zeta}}\left(\int_{0}^{T}\left(\bar{u}_{0}(t)\right)^{\frac{2}{2-\zeta}} d t\right)^{\frac{2-\zeta}{2}}\left\|u_{1}\right\|^{\zeta} \tau+M \tau \int_{0}^{T} \bar{u}_{0}(t) d t \\
& \left.-\frac{\beta}{\omega+1} \tau^{\omega+1} \int_{0}^{T}\left(\bar{u}_{0}(t)\right)^{\omega+1} d t\right] \\
\leq & \max _{\tau \in[0, \infty)}\left[\frac{1}{2} \tau^{2}+\sum_{j=1}^{n} \bar{\beta}_{j} \tau^{\omega_{j}+1}+\left(\sum_{j=1}^{n} \bar{M}_{j}+\bar{M}\right) \tau+\bar{\lambda} K^{\zeta} \tau-\bar{\beta} \tau^{\omega+1}\right],
\end{aligned}
$$

where $\bar{\beta}_{j}=\frac{\beta_{j}\left(\bar{u}_{0}\left(t_{j}\right)\right)^{\omega_{j}+1}}{\omega_{j}+1}, \bar{M}_{j}=M_{j} \bar{u}_{0}\left(t_{j}\right), \bar{M}=M \int_{0}^{T} \bar{u}_{0}(t) d t, \bar{\beta}=\frac{\beta}{\omega+1} \int_{0}^{T}\left(\bar{u}_{0}(t)\right)^{\omega+1} d t=$ $\frac{\beta(\omega+3) T}{2(\omega+1)(\omega+2) \Lambda_{0}^{\omega+1}}, \bar{\lambda}=\frac{\lambda}{\eta_{1}^{\zeta}}\left(\int_{0}^{T}\left(\bar{u}_{0}(t)\right)^{\frac{2}{2-\zeta}} d t\right)^{\frac{2-\zeta}{2}}=\frac{\lambda}{\eta_{1}^{\zeta} \Lambda_{0}}\left(\frac{(3-\zeta) T}{4-\zeta}\right)^{\frac{2-\zeta}{2}}$ and the relations

$$
\begin{aligned}
\left.\left.\int_{0}^{T} \bar{u}_{0}(t)\right|_{0} ^{c} D_{t}^{\alpha} u_{1}(t)\right|^{\zeta} d t & \leq\left(\int_{0}^{T}\left(\bar{u}_{0}(t)\right)^{\left.\frac{2}{2-\zeta} d t\right)^{\frac{2-\zeta}{2}}\left\|u_{1}\right\|_{\alpha, 2}^{\zeta}}\right. \\
& \leq\left(\int_{0}^{T}\left(\bar{u}_{0}(t)\right)^{\frac{2}{2-\zeta}} d t\right)^{\frac{2-\zeta}{2}} \frac{1}{\eta_{1}^{\zeta}}\left\|u_{1}\right\|^{\zeta}
\end{aligned}
$$

and $\left\|u_{1}\right\| \leq K$ are used.

Applying the Young inequality, we have

$$
\bar{\lambda} K^{\zeta} \tau \leq \frac{1}{q}\left(\frac{1}{\varepsilon_{0}} \bar{\lambda} \tau\right)^{q}+\frac{1}{p}\left(\varepsilon_{0} K^{\zeta}\right)^{p}=\frac{2-\zeta}{2}(\bar{\lambda} \tau)^{\frac{2}{2-\zeta}}\left(\frac{8 \mu \zeta}{\mu-2}\right)^{\frac{\zeta}{2-\zeta}}+\frac{\mu-2}{16 \mu} K^{2}
$$

where $p=\frac{2}{\zeta}, q=\frac{2}{2-\zeta}$ and $\varepsilon_{0}=\left(\frac{\mu-2}{8 \mu \zeta}\right)^{\frac{\zeta}{2}}$.

Denote $\bar{r}=\frac{2-\zeta}{2} \bar{\lambda}^{\frac{2}{2-\zeta}}\left(\frac{8 \mu \zeta}{\mu-2}\right)^{\frac{\zeta}{\mu-2}}$. Then from (3.4), it follows that

$$
\phi_{u_{1}}\left(u_{2}\right) \leq \max _{\tau \in[0, \infty)}\left[\frac{1}{2} \tau^{2}+\sum_{j=1}^{n} \bar{\beta}_{j} \tau^{\omega_{j}+1}+\left(\sum_{j=1}^{n} \bar{M}_{j}+\bar{M}\right) \tau+\bar{r} \tau^{\frac{2}{2-\zeta}}-\bar{\beta} \tau^{\omega+1}+\frac{\mu-2}{16 \mu} K^{2}\right]
$$

Let $H(\tau)=\frac{1}{2} \tau^{2}+\sum_{j=1}^{n} \bar{\beta}_{j} \tau^{\omega_{j}+1}+\left(\sum_{j=1}^{n} \bar{M}_{j}+\bar{M}\right) \tau+\bar{r} \tau^{\frac{2}{2-\zeta}}-\bar{\beta} \tau^{\omega+1}$. Then

$$
\phi_{u_{1}}\left(u_{2}\right) \leq \max _{\tau \in[0, \infty)} H(\tau)+\frac{\mu-2}{16 \mu} K^{2} .
$$


(1) If $0 \leq \tau \leq 1$, then

$$
H(\tau) \leq \frac{1}{2}+\sum_{j=1}^{n} \bar{\beta}_{j}+\sum_{j=1}^{n} \bar{M}_{j}+\bar{M}+\bar{r}:=A,
$$

noting that $0<\zeta, \omega_{j}<1$

(2) If $1 \leq \tau<\infty$, then

$$
H(\tau) \leq\left(\frac{1}{2}+\sum_{j=1}^{n} \bar{\beta}_{j}+\sum_{j=1}^{n} \bar{M}_{j}+\bar{r}\right) \tau^{2}-\bar{\beta} \tau^{\omega+1}=A \tau^{2}-\bar{\beta} \tau^{\omega+1}:=\Phi(\tau),
$$

noting that $0<\zeta, \omega_{j}<1$.

By $\Phi^{\prime}(\tau)=2 A \tau-\bar{\beta}(\omega+1) \tau^{\omega}$, if let $\Phi^{\prime}(\tau)=0$, then $\tau=\bar{\tau}:=\left(\frac{2 A}{\bar{\beta}(\omega+1)}\right) \frac{1}{\omega-1}$ and $\Phi(\bar{\tau})=$ $\max _{\tau \in[1, \infty)} \Phi(\tau)=\frac{(\omega-1) A}{\omega+1}\left(\frac{2 A}{\bar{\beta}(\omega+1)}\right)^{\frac{2}{\omega-1}}:=B$.

Thus, summing up the above discussions (1) and (2), we always have

$$
\Phi(\tau) \leq \max \{A, B\}:=C, \quad \tau \in[0, \infty)
$$

and therefore

$$
\phi_{u_{1}}\left(u_{2}\right) \leq C+\frac{\mu-2}{16 \mu} K^{2}
$$

On the other hand, because

$$
\begin{aligned}
& \mu \phi_{u_{1}}\left(u_{2}\right)-\phi_{u_{1}}^{\prime}\left(u_{2}\right) u_{2} \\
& =\left(\frac{\mu}{2}-1\right)\left\|u_{2}\right\|^{2}+\int_{0}^{T}\left[f\left(t, u_{2}(t),{ }_{0}^{c} D_{t}^{\alpha} u_{1}(t)\right) u_{2}(t)-\mu F\left(t, u_{2},{ }_{0}^{c} D_{t}^{\alpha} u_{1}\right)\right] d t \\
& \quad+\sum_{j=1}^{n}\left(\mu \int_{0}^{u_{2}\left(t_{j}\right)} I_{j}(s) d s-I_{j}\left(u_{2}\left(t_{j}\right)\right) u_{2}\left(t_{j}\right)\right)
\end{aligned}
$$

and $\phi_{u_{1}}^{\prime}\left(u_{2}\right)=0, \phi_{u_{1}}\left(u_{2}\right) \leq C+\frac{\mu-2}{16 \mu} K^{2}$, by $\left(H_{3}\right)$ and by a discussion similar to (3.2), we have

$$
\begin{aligned}
& \left(\frac{\mu}{2}-1\right)\left\|u_{2}\right\|^{2} \\
& \leq \mu \phi_{u_{1}}\left(u_{2}\right)+\int_{0}^{T}\left[\mu F\left(t, u_{2}(t),{ }_{0}^{c} D_{t}^{\alpha} u_{1}(t)\right)-f\left(t, u_{2}(t),{ }_{0}^{c} D_{t}^{\alpha} u_{1}(t)\right) u_{2}(t)\right] d t \\
& \quad+\sum_{j=1}^{n}\left(I_{j}\left(u_{2}\left(t_{j}\right)\right) u_{2}\left(t_{j}\right)-\mu \int_{0}^{u_{2}\left(t_{j}\right)} I_{j}(s) d s\right) \\
& \leq \mu\left(C+\frac{\mu-2}{16 \mu} K^{2}\right)+\bar{l}\left\|u_{2}\right\|^{\sigma}+\sum_{j=1}^{n} \bar{l}_{j}\left\|u_{2}\right\|^{\sigma_{j}}+\sum_{j=1}^{n} d_{j}+d T+m \int_{0}^{T}\left|{ }_{0}^{c} D_{t}^{\alpha} u_{1}(t)\right|^{\tau} d \tau \\
& \leq \mu C+\frac{\mu-2}{16} K^{2}+\bar{l}\left\|u_{2}\right\|^{\sigma}+\sum_{j=1}^{n} \bar{l}_{j}\left\|u_{2}\right\|^{\sigma_{j}}+\bar{d}+\bar{m} K^{\tau}
\end{aligned}
$$


where $\bar{d}=\sum_{j=1}^{n} d_{j}+d T, \bar{m}=T^{\frac{2-\tau}{\tau}} \eta_{1}^{-\tau} m$ and the formulas

$$
\int_{0}^{T}\left|{ }_{0}^{c} D_{t}^{\alpha} u_{1}(t)\right|^{\tau} d \tau \leq T^{\frac{2-\tau}{2}}\left\|u_{1}\right\|_{\alpha, 2}^{\tau} \leq T^{\frac{2-\tau}{2}} \eta_{1}^{-1}\left\|u_{1}\right\|^{\tau} \leq T^{\frac{2-\tau}{2}} \eta_{1}^{-\tau} K^{\tau}
$$

are used.

By use of the Young inequality, similarly to (3.5), we get

$$
\begin{aligned}
& \bar{m} K^{\tau} \leq \frac{2-\tau}{2} \bar{m}^{\frac{2}{2-\tau}}\left(\frac{8 \tau}{\mu-2}\right)^{\frac{\tau}{2-\tau}}+\frac{\mu-2}{16} K^{2}=m^{*}+\frac{\mu-2}{16} K^{2}, \\
& \bar{l}\left\|u_{2}\right\|^{\sigma} \leq \frac{2-\sigma}{2} \bar{l}^{\frac{2}{2-\sigma}}\left(\frac{8 \sigma}{\mu-2}\right)^{\frac{\sigma}{2-\sigma}}+\frac{\mu-2}{16}\left\|u_{2}\right\|^{2}=l^{*}+\frac{\mu-2}{16}\left\|u_{2}\right\|^{2}, \\
& \bar{l}_{j}\left\|u_{2}\right\|^{\sigma_{j}} \leq \frac{2-\sigma_{j}}{2} \bar{l}_{j}^{\frac{2}{2-\sigma_{j}}}\left(\frac{8 n \sigma_{j}}{\mu-2}\right)^{\frac{\sigma_{j}}{2-\sigma_{j}}}+\frac{\mu-2}{16 n}\left\|u_{2}\right\|^{2}=l_{j}^{*}+\frac{\mu-2}{16 n}\left\|u_{2}\right\|^{2},
\end{aligned}
$$

where $m^{*}=\frac{2-\tau}{2} \bar{m} \frac{2}{2-\tau}\left(\frac{8 \tau}{\mu-2}\right)^{\frac{\tau}{2-\tau}}, l^{*}=\frac{2-\sigma}{2} \bar{l} \frac{2}{2-\sigma}\left(\frac{8 \sigma}{\mu-2}\right) \frac{\sigma}{2-\sigma}, l_{j}^{*}=\frac{2-\sigma_{j}}{2} \bar{l}_{j}^{\frac{2}{2-\sigma_{j}}}\left(\frac{8 n \sigma_{j}}{\mu-2}\right)^{\frac{\sigma_{j}}{2-\sigma_{j}}}$.

So, it follows from (3.6) that

$$
\begin{aligned}
& \frac{\mu-2}{2}\left\|u_{2}\right\|^{2} \leq \mu C+m^{*}+l^{*}+\sum_{j=1}^{n} l_{j}^{*}+\bar{d}+\frac{\mu-2}{4} K^{2}, \\
& \left\|u_{2}\right\|^{2} \leq \frac{2}{\mu-2}\left(\mu C+m^{*}+l^{*}+\sum_{j=1}^{n} l_{j}^{*}+\bar{d}\right)+\frac{1}{2} K^{2} .
\end{aligned}
$$

Take $K=\sqrt{2 E}$, where $E=\frac{2}{\mu-2}\left(\mu C+m^{*}+l^{*}+\sum_{j=1}^{n} l_{j}^{*}+\bar{d}\right)$, then $\left\|u_{2}\right\|^{2} \leq K^{2}$, i.e., $\left\|u_{2}\right\| \leq K$.

Assume that $\left\|u_{n-1}\right\| \leq K$, by the same process as above, we can deduce that $\left\|u_{n}\right\| \leq K$. Thus for all $n \in \mathbb{N},\left\|u_{n}\right\| \leq K$.

Step 5. We show that $\left\{u_{n}\right\}$ converges to $u^{*} \in X^{\alpha}$. In fact, by the proof in Step 4 , we know that $\left\|u_{n}\right\| \leq K$. It follows from Lemma 2.2 and Lemma 2.6 that

$$
\left\|u_{n}\right\|_{\infty} \leq \frac{T^{\alpha-1 / 2}}{\eta_{1} \Gamma(\alpha) \sqrt{2 \alpha-1}}\left\|u_{n}\right\| \leq \frac{T^{\alpha-1 / 2}}{\eta_{1} \Gamma(\alpha) \sqrt{2 \alpha-1}} K=K_{1},
$$

and therefore, by $\left(H_{4}\right)$ we have

$$
\begin{aligned}
& \left|\int_{0}^{T}\left[f\left(t, u_{n+1}(t),{ }_{0}^{c} D_{t}^{\alpha} u_{n}(t)\right)-f\left(t, u_{n}(t),{ }_{0}^{c} D_{t}^{\alpha} u_{n-1}(t)\right)\left(u_{n+1}(t)-u_{n}(t)\right)\right] d t\right| \\
& \quad \leq \int_{0}^{T}\left[p(t)\left|u_{n+1}(t)-u_{n}(t)\right|^{2}+q(t)\left|{ }_{0}^{c} D_{t}^{\alpha}\left(u_{n}-u_{n-1}\right)(t) \| u_{n+1}(t)-u_{n}(t)\right|\right] d t \\
& \leq\left\|u_{n+1}(t)-u_{n}(t)\right\|_{\infty}\left(\int_{0}^{T} p(t)\left|u_{n+1}(t)-u_{n}(t)\right| d t+\int_{0}^{T} q(t)\left|{ }_{0}^{c} D_{t}^{\alpha}\left(u_{n}-u_{n-1}\right)(t)\right| d t\right) \\
& \leq\left\|u_{n+1}(t)-u_{n}(t)\right\|_{\infty}\left(\|p\|_{2} \cdot\left\|u_{n+1}-u_{n}\right\|_{2}+\|q\|_{2} \cdot\|\|_{0}^{c} D_{t}^{\alpha}\left(u_{n}-u_{n-1}\right) \|_{2}\right) \\
& \leq \frac{T^{2 \alpha-1 / 2}}{\Gamma(\alpha) \Gamma(\alpha+1) \sqrt{2 \alpha-1} \eta_{1}^{2}}\|p\|_{2} \cdot\left\|u_{n+1}-u_{n}\right\|^{2} \\
& \quad+\frac{T^{\alpha-1 / 2}}{\Gamma(\alpha) \sqrt{2 \alpha-1} \eta_{1}^{2}}\|q\|_{2} \cdot\left\|u_{n+1}-u_{n}\right\| \cdot\left\|u_{n}-u_{n-1}\right\|
\end{aligned}
$$


and

$$
\begin{aligned}
& \mid I_{j}\left(u_{n+1}\left(t_{j}\right)-I_{j}\left(u_{n}\left(t_{j}\right)\right)|| u_{n+1}\left(t_{j}\right)-u_{n}\left(t_{j}\right) \mid\right. \\
& \quad \leq a_{j}\left|u_{n+1}\left(t_{j}\right)-u_{n}\left(t_{j}\right)\right|^{2} \\
& \quad \leq \frac{T^{2 \alpha-1} a_{j}}{(\Gamma(\alpha))^{2}(2 \alpha-1) \eta_{1}^{2}}\left\|u_{n+1}-u_{n}\right\|^{2}, \quad j=1,2, \ldots, n .
\end{aligned}
$$

On the other hand, because $\phi_{u_{n}}^{\prime}\left(u_{n+1}\right)\left(u_{n+1}-u_{n}\right)=0, \phi_{u_{n-1}}^{\prime}\left(u_{n}\right)\left(u_{n+1}-u_{n}\right)=0$, in view of (3.7)-(3.8) and the following relation:

$$
\begin{aligned}
& \left(\phi_{u_{n}}^{\prime}\left(u_{n+1}\right)-\phi_{u_{n-1}}^{\prime}\left(u_{n}\right)\right)\left(u_{n+1}-u_{n}\right) \\
& =\left\|u_{n+1}-u_{n}\right\|^{2}+\sum_{j=1}^{n}\left[I_{j}\left(u_{n+1}\left(t_{j}\right)\right)-I_{j}\left(u_{n}\left(t_{j}\right)\right)\right]\left(u_{n+1}\left(t_{j}\right)-u_{n}\left(t_{j}\right)\right) \\
& \quad-\int_{0}^{T}\left[f\left(t, u_{n+1}(t),{ }_{0}^{c} D_{t}^{\alpha} u_{n}(t)\right)-f\left(t, u_{n}(t),{ }_{0}^{c} D_{t}^{\alpha} u_{n-1}(t)\right)\right]\left(u_{n+1}(t)-u_{n}(t)\right) d t,
\end{aligned}
$$

we obtain

$$
\begin{aligned}
\| u_{n+1}- & u_{n} \|^{2} \\
= & \int_{0}^{T}\left[f\left(t, u_{n+1}(t),{ }_{0}^{c} D_{t}^{\alpha} u_{n}(t)\right)-f\left(t, u_{n}(t),{ }_{0}^{c} D_{t}^{\alpha} u_{n-1}(t)\right)\right]\left(u_{n+1}(t)-u_{n}(t)\right) d t \\
& \quad-\sum_{j=1}^{n}\left[I_{j}\left(u_{n+1}\left(t_{j}\right)\right)-I_{j}\left(u_{n}\left(t_{j}\right)\right)\right]\left(u_{n+1}\left(t_{j}\right)-u_{n}\left(t_{j}\right)\right) \\
\leq & \left(\frac{T^{2 \alpha-1 / 2}}{\Gamma(\alpha) \Gamma(\alpha+1) \sqrt{2 \alpha-1} \eta_{1}^{2}}\|p\|_{L^{2}}+\frac{T^{2 \alpha-1}}{(\Gamma(\alpha))^{2}(2 \alpha-1) \eta_{1}^{2}} \sum_{j=1}^{n} a_{j}\right)\left\|u_{n+1}-u_{n}\right\|^{2} \\
& +\frac{T^{\alpha-1 / 2}}{\Gamma(\alpha) \sqrt{2 \alpha-1} \eta_{1}^{2}}\|q\|_{2}\left\|u_{n+1}-u_{n}\right\| \cdot\left\|u_{n}-u_{n-1}\right\| \\
= & P_{0}\left\|u_{n+1}-u_{n}\right\|^{2}+Q_{0}\left\|u_{n+1}-u_{n}\right\| \cdot\left\|u_{n}-u_{n-1}\right\|,
\end{aligned}
$$

where $P_{0}=\frac{T^{2 \alpha-1 / 2}}{\Gamma(\alpha) \Gamma(\alpha+1) \sqrt{2 \alpha-1} \eta_{1}^{2}}\|p\|_{2}+\frac{T^{2 \alpha-1}}{(\Gamma(\alpha))^{2}(2 \alpha-1) \eta_{1}^{2}} \sum_{j=1}^{n} a_{j}, Q_{0}=\frac{T^{\alpha-1 / 2}}{\Gamma(\alpha) \sqrt{2 \alpha-1} \eta_{1}^{2}}\|q\|_{2}$.

Hence

$$
\left\|u_{n+1}-u_{n}\right\| \leq P_{0}\left\|u_{n+1}-u_{n}\right\|+Q_{0}\left\|u_{n}-u_{n-1}\right\|
$$

By the assumption $P_{0}<1$, we have

$$
\left\|u_{n+1}-u_{n}\right\| \leq \frac{Q_{0}}{1-P_{0}}\left\|u_{n}-u_{n-1}\right\|
$$

Owing to the assumption $0 \leq \frac{Q_{0}}{1-P_{0}}<1$, it follows from (3.9) that the sequence $\left\{u_{n}\right\}$ converges to a point $u^{*} \in X^{\alpha}$, and so, $u_{n} \rightarrow u$ in $C[0, T]$. Thus, $\left\|u^{*}\right\| \leq K$ and $\left\|u^{*}\right\|_{\infty} \leq K_{1}$. 
Finally, because

$$
\begin{aligned}
& \int_{0}^{T}\left[\left|{ }_{0}^{c} D_{t}^{\alpha}\left(u_{n}-u^{*}\right)(t)\left\|{ }_{0}^{c} D_{t}^{\alpha} v(t)|+| a(t)\right\| u_{n}(t)-u^{*}(t)\right|\right]|v(t)| d t \\
& \quad \leq\left\|{ }_{0}^{c} D_{t}^{\alpha}\left(u_{n}-u^{*}\right)\right\|_{2} \cdot\left\|{ }_{0}^{c} D_{t}^{\alpha} v\right\|_{2}+\|a\|_{\infty}\left\|u_{n}-u^{*}\right\|_{2} \cdot\|v\|_{2} \\
& \leq \frac{1}{\eta_{1}^{2}}\left\|u_{n}-u^{*}\right\| \cdot\|v\|+\|a\|_{\infty} \frac{T^{2 \alpha}}{\Gamma^{2}(\alpha+1) \eta_{1}^{2}}\left\|u_{n}-u^{*}\right\| \cdot\|v\|
\end{aligned}
$$

for all $v \in X^{\alpha}$, the convergence $u_{n} \rightarrow u^{*}$ implies

$$
\begin{aligned}
& \int_{0}^{T}\left[{ }_{0}^{c} D_{t}^{\alpha} u_{n}(t) \cdot{ }_{0}^{c} D_{t}^{\alpha} v(t)+a(t) u_{n}(t) v(t)\right] d t \\
& \quad \rightarrow \int_{0}^{T}\left[{ }_{0}^{c} D_{t}^{\alpha} u^{*}(t) \cdot{ }_{0}^{c} D_{t}^{\alpha} v(t)+a(t) u^{*}(t) v(t)\right] d t
\end{aligned}
$$

as $n \rightarrow \infty$. Also, obviously,

$$
\sum_{j=1}^{n} I_{j}\left(u_{n}\left(t_{j}\right)\right) v\left(t_{j}\right) \rightarrow \sum_{j=1}^{n} I_{j}\left(u^{*}\left(t_{j}\right)\right) v\left(t_{j}\right)
$$

as $n \rightarrow \infty$. By $\left(H_{4}\right)$, observing

$$
\begin{aligned}
& \left|\int_{0}^{T}\left[f\left(t, u_{n}(t),{ }_{0}^{c} D_{t}^{\alpha} u_{n-1}(t)\right)-f\left(t, u^{*}(t),{ }_{0}^{c} D_{t}^{\alpha} u^{*}(t)\right)\right] v(t) d t\right| \\
& \quad \leq \int_{0}^{T}\left[p(t)\left|u_{n}(t)-u^{*}(t)\right|+q(t)\left|{ }_{0}^{c} D_{t}^{\alpha}\left(u_{n-1}-u^{*}\right)(t)\right| \cdot|v(t)|\right] d t \\
& \quad \leq\|v\|_{\infty}\left(\|p\|_{2} \cdot\left\|u_{n}-u^{*}\right\|_{2}+\|q\|_{2} \cdot\left\|{ }_{0}^{c} D_{t}^{\alpha}\left(u_{n-1}-u^{*}\right)\right\|_{2}\right) \\
& \quad \leq\|v\|_{\infty}\left(\|p\|_{2} \frac{T^{\alpha}}{\Gamma(\alpha+1) \eta_{1}}\left\|u_{n}-u^{*}\right\|+\|q\|_{2} \frac{1}{\eta_{1}}\left\|u_{n-1}-u^{*}\right\|\right),
\end{aligned}
$$

we know that

$$
\int_{0}^{T} f\left(t, u_{n}(t),{ }_{0}^{c} D_{t}^{\alpha} u_{n-1}(t)\right) v(t) d t \rightarrow \int_{0}^{T} f\left(t, u^{*}(t),{ }_{0}^{c} D_{t}^{\alpha} u^{*}(t)\right) v(t) d t
$$

as $n \rightarrow \infty$. Also, by (2.5), the fact that $\phi_{u_{n-1}}^{\prime}\left(u_{n}\right) v=0$ means that

$$
\begin{aligned}
0= & \int_{0}^{T}\left[{ }_{0}^{c} D_{t}^{\alpha} u_{n}(t) \cdot{ }_{0}^{c} D_{t}^{\alpha} v(t)+a(t) u_{n}(t) v(t)\right] d t \\
& +\sum_{j=1}^{n} I_{j}\left(u_{n}\left(t_{j}\right)\right) v\left(t_{j}\right)-\int_{0}^{T} f\left(t, u_{n}(t),{ }_{0}^{c} D_{t}^{\alpha} u_{n-1}(t)\right) v(t) d t
\end{aligned}
$$

for all $v \in X^{\alpha}$. The above equality combined with (3.10)-(3.12) implies

$$
\begin{aligned}
0= & \int_{0}^{T}\left[{ }_{0}^{c} D_{t}^{\alpha} u^{*}(t) \cdot{ }_{0}^{c} D_{t}^{\alpha} v(t)+a(t) u^{*}(t) v(t)\right] d t \\
& +\sum_{j=1}^{n} I_{j}\left(u^{*}\left(t_{j}\right)\right) v\left(t_{j}\right)-\int_{0}^{T} f\left(t, u^{*}(t),{ }_{0}^{c} D_{t}^{\alpha} u^{*}(t)\right) v(t) d t
\end{aligned}
$$


for all $v \in X^{\alpha}$, i.e., $\phi_{u^{*}}^{\prime}\left(u^{*}\right) v=0$. This means that $u^{*}$ is a weak solution of BVP (1.2). Similarly, we can prove that $\lim _{x \rightarrow \infty} \phi_{u_{n-1}}\left(u_{n}\right)=\phi_{u^{*}}\left(u^{*}\right)$. Because $\phi_{u_{n-1}}\left(u_{n}\right) \geq \alpha_{1}>0$, we conclude that $\phi_{u^{*}}\left(u^{*}\right) \geq \alpha_{1}>0$. This means that $u^{*}$ is a nontrivial classical solution of BVP (1.2) taking into account Lemma 2.4 .

Example 3.1 Consider the following BVP:

$$
\left\{\begin{array}{l}
{ }_{t} D_{1}^{\frac{2}{3}}\left({ }_{0}^{c} D_{t}^{\frac{2}{3}} u(t)\right)+t u(t)=f\left(t, u(t),{ }_{0}^{c} D_{t}^{\frac{2}{3}} u(t)\right), \quad t \neq \frac{1}{2}, t \in[0,1] \\
\Delta\left({ }_{t} D_{1}^{-\frac{1}{3}}\left({ }_{0}^{c} D_{t}^{\frac{2}{3}} u(t)\right)\right)\left(\frac{1}{2}\right)=I_{1}\left(u\left(\frac{1}{2}\right)\right), \\
u(0)=u(1)=0
\end{array}\right.
$$

with respect to BVP (1.2), where $\alpha=\frac{2}{3}, j=1, T=1, a(t)=t$, and functions $f, I_{1}$ have the following forms, respectively:

$$
f(t, x, y)=b e^{-t} x^{5}+c_{1} t^{2} x^{3}(\sin y)^{\frac{4}{3}}-c_{2}(\cos t) h(x) g(y), \quad t \in[0,1], x, y \in \mathbb{R},
$$

where $b>0, c_{1}, c_{2}>0$,

$$
h(x)=\left\{\begin{array}{ll}
0, & x \leq 0, \\
x, & 0 \leq x \leq 1, \\
1, & 1 \leq x<\infty,
\end{array} \quad g(y)= \begin{cases}0, & y \leq 0 \\
y^{3}, & 0 \leq y \leq 1 \\
y^{\frac{1}{3}}, & 1 \leq y \leq L_{0} \\
L_{0}^{\frac{1}{3}}, & L_{0} \leq y<\infty\end{cases}\right.
$$

where the constant $L_{0}>0$, and

$$
I_{1}(s)=-b_{1} s|s|, \quad s \in \mathbb{R}, b_{1}>0 .
$$

We verify that the conditions hold corresponding to $\left(H_{1}\right)-\left(H_{4}\right)$ in Theorem 3.1.

$\left(H_{1}\right) f(t, x, y) \leq b|x|^{5}+c_{1}|x|^{3}|y|^{\frac{4}{3}}, t \in[0,1], x, y \in \mathbb{R} ; I_{1}(s) \geq-b_{1}|s|^{2}, s \in \mathbb{R}$.

$\left(H_{2}\right) \quad x f(t, x, y)-3 F(t, x, y) \geq 0, t \in[0,1], x, y \in \mathbb{R} ; 3 \int_{0}^{u} I_{1}(s) d s-I_{1}(u) u \geq 0, x \in \mathbb{R}$.

$\left(H_{3}\right) f(t, x, y) \geq b x^{5}-c_{2}|y|^{\frac{1}{3}}, t \in[0,1], x \geq 0, y \in \mathbb{R} ; I_{1}(s) \leq 0, s \geq 0$.

$\left(H_{4}\right)\left|f\left(t, x_{2}, y_{2}\right)-f\left(t, x_{1}, y_{1}\right)\right| \leq p(t)\left|x_{2}-x_{1}\right|+q(t)\left|y_{2}-y_{1}\right|, t \in[0,1], y_{1}, y_{2} \in \mathbb{R}, x_{1}, x_{2} \in$ $\left[-K_{1}, K_{2}\right]$ and

$$
\left|I_{1}(x)-I_{1}(y)\right| \leq 2 b_{1} K_{1}|x-y|, \quad \forall x, y \in\left[-K_{1}, K_{1}\right]
$$

$$
\text { where } p(t)=5 b K_{1}^{4} e^{-t}+3 c_{1} K_{1}^{2} t^{2}+c_{2} L_{0}^{\frac{1}{3}} \cos t, q(t)=\frac{4}{3} c_{1} K_{1}^{3} t^{2}+3 c_{2} \cos t, t \in[0,1] \text {. }
$$

Compared with the conditions in Theorem 3.1, here $\eta_{1}=3 / 4, b=\beta>0, \omega=\gamma=5, \xi=3$, $\theta=4 / 3, \gamma_{1}=2, \mu=3, \lambda=c_{2}>0, \zeta=1 / 3, a_{1}=2 b_{1} K_{1}, l=m=d=L=M=J=0, M_{1}=\beta_{1}=$ $d_{1}=l_{1}=0$, and so $\bar{M}=\bar{d}=\bar{m}=\bar{l}=0, \bar{l}_{1}=\bar{\beta}_{1}=\bar{M}_{1}=0$, as well as $m^{*}=l^{*}=l_{1}^{*}=0$.

By direct computation, we know that $\bar{\beta}=\frac{2 b}{21 \Lambda_{0}^{6}}, \bar{r}=\frac{40}{33}\left(\frac{4}{3}\right)^{\frac{2}{5}}\left(\frac{c_{2}}{\Lambda_{0}}\right)^{\frac{6}{5}}$ and $A=\frac{1}{2}+\bar{r}, B=$ $\frac{2 A}{3}\left(\frac{7 A}{2 b}\right)^{\frac{1}{2}} \Lambda_{0}^{3}, C=\max \{A, B\}, K=\sqrt{12 C}$ and $K_{1}=\frac{4 \sqrt{3}}{3 \Gamma\left(\frac{2}{3}\right)} K, P_{0}=\frac{16}{3 \Gamma\left(\frac{2}{3}\right) \Gamma\left(\frac{5}{3}\right)}\|p\|_{2}+\frac{16}{3\left(\Gamma\left(\frac{2}{3}\right)\right)^{2}} a_{1}$, 


$$
\begin{aligned}
& Q_{0}=\frac{16 \sqrt{3}}{9 \Gamma\left(\frac{2}{3}\right)}\|q\|_{2} \\
& \qquad p \|_{2}^{2}= \frac{9}{5} c_{1}^{2} K_{1}^{4}+\left(\frac{1}{4} \sin 2+\frac{1}{2}\right) c_{2}^{2} L_{0}^{\frac{1}{3}}+30 b_{1} c_{1} K_{1}^{6}\left(2-\frac{5}{e}\right)+b c_{1} c_{2} K_{1}^{2} L_{0}^{\frac{1}{3}}(2 \cos 1-\sin 1) \\
&+5 b c_{2} L_{0}^{\frac{1}{3}} K^{4}\left(1+\frac{\sin 1-\cos 1}{e}\right)+\frac{25}{2} b^{2} K^{4}\left(1-e^{-2}\right) \\
&\|q\|_{2}^{2}= \frac{16}{45} c_{1}^{2} K_{1}^{6}+9 c_{1}^{2}\left(\frac{1}{4} \sin 2+\frac{1}{2}\right)+8 c_{1} K_{1}^{3}(2 \cos 1-\sin 1)
\end{aligned}
$$

Obviously, we can choose the suitably small constants $b, c_{1}, c_{2}$ such that $P_{0}<1$ and $Q_{0}<$ $1-P_{0}$ and therefore it follows from Theorem 3.1 that BVP (3.13) has at least one nontrivial classical solution.

\section{Competing interests}

The authors declare that they have no competing interests.

\section{Authors' contributions}

The authors declare that they carried out all the work in this manuscript and read and approved the final manuscript.

\section{Acknowledgements}

The authors sincerely thank the anonymous referees for their valuable suggestions and comments which have greatly helped improve this article. This research is supported by the National Nature Science Foundation of China under grant 11601139.

Received: 26 September 2016 Accepted: 27 January 2017 Published online: 08 February 2017

\section{References}

1. Diethelm, K, Freed, AD: On the solution of nonlinear fractional order differential equations used in the modeling of viscoelasticity. In: Keil, F, Mackens, W, Voss, H, Werther, J (eds.) Scientific Computing in Chemical Engineering II Computational Fluid Dynamics, Reaction Engineering and Molecular Properties, pp. 217-224. Springer, Heidelberg (1999)

2. Lundstrom, BN, Higgs, MH, Spain, WJ, Fairhall, AL: Fractional differentiation by neocortical pyramidal neurons. Nat. Neurosci. 11, 1335-1342 (2008)

3. Glockle, WG, Nonnenmacher, TF: A fractional calculus approach of self-similar protein dynamics. Biophys. J. 68, 46-53 (1995)

4. Hilfer, R: Applications of Fractional Calculus in Physics. World Scientific, Singapore (2000)

5. Mainardi, F: Fractional calculus: some basic problem in continuum and statistical mechanics. In: Carpinteri, A, Mainardi, F (eds.) Fractals and Fractional Calculus in Continuum Mechanics, pp. 290-349. Springer, Wien (1997)

6. Kilbas, AA, Srivastava, HM, Trujillo, JJ: Theory and Applications of Fractional Differential Equations. North-Holland Mathematics Studies, vol. 204. Elsevier, Amsterdam (2006)

7. Jiao, F, Zhou, Y: Existence results for fractional boundary value problems via critical point theory. Int. J. Bifurc. Chaos $22,1250086(2012)$

8. Wu, X, Zhang, Z: Solutions for perturbed fractional Hamiltonian systems without coercive conditions. Bound. Value Probl. 2015, 149 (2015)

9. Bai, C: Infinitely many solutions for a perturbed nonlinear fractional boundary-value problem. Electron. J. Differ. Equ. 2013, $136(2013)$

10. Zhao, Y, Chen, H, Qin, B: Multiple solutions for a coupled system of nonlinear fractional differential equations via variational methods. Appl. Math. Comput. 257, 417-427 (2015)

11. Zhao, Y, Chen, H, Zhang, Q: Infinitely many solutions for fractional differential system via variational method. J. Appl. Math. Comput. 50, 589-609 (2016)

12. Zhang, Z, Li, J: Variational approach to solutions for a class of fractional boundary value problems. Electron. J. Qual. Theory Differ. Equ. 2015, 11 (2015)

13. Chai, G: Infinitely many solutions for nonlinear fractional boundary value problems via variational methods. Adv. Differ. Equ. 2016, 213 (2016)

14. Hu, Z, Liu, W, Chen, T: The existence of a ground state solution for a class of fractional differential equation with p-Laplacian operator. Bound. Value Probl. 2016, 45 (2016)

15. Chen, T, Liu, W: Solvability of fractional boundary value problem with $p$-Laplacian via critical point theory. Bound. Value Probl. 2016, 75 (2016)

16. Bonanno, G, Rodríguez-López, R, Tersian, S: Existence of solutions to boundary value problem for impulsive fractional differential equations. Fract. Calc. Appl. Anal. 17(3), 717-744 (2014)

17. Rodríguez-López, R, Tersian, S: Multiple solutions to boundary value problem for impulsive fractional differential equations. Fract. Calc. Appl. Anal. 17(4), 1016-1038 (2014)

18. Nyamoradi, N, Rodríguez-López, R: On boundary value problems for impulsive fractional differential equations. Appl. Math. Comput. 271, 874-892 (2015) 
19. Lakshmikantham, V, Bainov, D, Simeonov, P: Impulsive Differential Equations and Inclusions. World Scientific, Singapore (1989)

20. Bonanno, G, Di Bella, B, Henderson, J: Existence of solutions to second-order boundary-value problems with small perturbations of impulses. Electron. J. Differ. Equ. 2013, 126 (2013)

21. Xiao, J, Nieto, JJ: Variational approach to some damped Dirichlet nonlinear impulsive differential equations. J. Franklin Inst. 348, 369-377 (2011)

22. Zhou, J, Li, Y: Existence and multiplicity of solutions for some Dirichlet problems with impulse effects. Nonlinear Anal. TMA 71, 2856-2865 (2009)

23. Nieto, J, O'Regan, D: Variational approach to impulsive differential equations. Nonlinear Anal., Real World Appl. 10 680-690 (2009)

24. Mawhin, J, Willem, M: Critical Point Theory and Hamiltonian Systems. Springer, Berlin (1989)

25. Bartolo, P, Benci, V, Fortunato, D: Abstract critical point theorems and applications to some nonlinear problems with "strong" resonance at infinity. Nonlinear Anal. 7, 981-1012 (1983)

\section{Submit your manuscript to a SpringerOpen ${ }^{\circ}$} journal and benefit from:

- Convenient online submission

Rigorous peer review

- Immediate publication on acceptance

- Open access: articles freely available online

- High visibility within the field

- Retaining the copyright to your article 